\title{
KOSZUL RESOLUTIONS AND THE STEENROD ALGEBRA ${ }^{1}$
}

\author{
BY STEWART B. PRIDDY
}

Communicated by Franklin Peterson, October 24, 1969

1. Introduction. We outline a simple procedure for constructing resolutions for a large class of algebras including the Steenrod algebra and the universal enveloping algebras. It is classical that the cohomology groups of a Lie algebra may be computed using the Koszul resolution [2, Chapter $8, \S 7]$. This resolution is particularly nice because it is a small subcomplex of the bar resolution. Our resolutions are conceptually analogous and so we call them Koszul resolutions and the algebras for which they are defined Koszul algebras. For each such algebra $A$ we give (Theorem 3 ) an explicit differential algebra whose homology is the cohomology algebra $H^{*}(A)$. Our theory ( $\$ 3,(1),(2))$ subsumes May's generalization of the classical Koszul resolution and his resolution for a restricted Lie algebra in characteristic 2 [3]. In the (motivating) case of the Steenrod algebra we also retrieve $(\$ 3,(3))$ the $\Lambda$ algebra of Kan et al. [1].

A detailed treatment of our results will appear in [5]. The author wishes to thank Daniel M. Kan and especially J. Peter May for several conversations and to acknowledge a debt to [1] and [3].

2. Koszul algebras and resolutions. We define Koszul algebras, give examples (2.2) and then define Koszul resolutions in (2.3). By an algebra $A$ we shall always mean an augmented algebra, $F \stackrel{\rightarrow}{\rightarrow} A \stackrel{\dot{\rightarrow}}{\rightarrow}$, of finite type over a field $F$. A presentation of $A$ is an epimorphism of algebras $\alpha: T\left\{x_{i}\right\} \rightarrow A$, where $T\left\{x_{i}\right\}$ is the tensor algebra. Let $R=\operatorname{Ker}(\alpha)$ then $A \cong T\left\{x_{i}\right\} / R$. Now $A$ is said to be a pre-Koszul algebra if it admits a presentation $\alpha$ such that $R$ is the two sided ideal generated by elements of the form $\sum_{i} f_{i} x_{i}+\sum_{j, k} f_{j, k} x_{j} x_{k}$ where $f_{i}$ and $f_{j, k}$ are in $F$. Let $\alpha\left(x_{i}\right)=a_{i}$. If $\left\{a_{i}\right\}$ are linearly independent then $\left\{a_{i}\right\}$ is called a pre-Koszul set of generators for $A$ and $\alpha$ is called a pre-Koszul presentation. Note that $A$ is defined by relations of the form

$$
\sum_{i} f_{i} a_{i}+\sum_{j, k} f_{j, k} a_{j} a_{k}=0
$$

A pre-Koszul algebra is said to be homogeneous if each $f_{i}=0$ in each relation (2.1). Now $T\left\{x_{i}\right\}$ is filtered with $F_{p} T\left\{x_{i}\right\}, p \geqq 0$, spanned by all

AMS Subject Classifications. Primary 1820; Secondary 5534.

Key Words and Phrases. Koszul resolutions, bar and cobar resolutions, cohomology of the Steenrod algebra, cohomology of Lie algebras.

1 This research was supported in part by NSF GP-8217. 
monomials of length $\leqq p$, with 1 having length 0 . If $\alpha: T\left\{x_{i}\right\} \rightarrow A$ is a presentation, define $F_{p} A=\alpha\left(F_{p} T\left\{x_{i}\right\}\right)$ and $E_{p, q}^{0} A=\left(F_{p} A / F_{p-1} A\right)_{p+q}$; if $\alpha$ is a pre-Koszul presentation then $E^{0} A$ is called the associated homogeneous pre-Koszul algebra of $A$. Note that $E^{0} A$ is generated by images $b_{i}$ of $a_{i}$ in $E_{1, *}^{0} A$ and is defined by the relations $\sum_{j, k} f_{j, k} b_{j} b_{k}$ $=0$.

Let $A$ be a homogeneous pre-Koszul algebra with generators $\left\{a_{i}\right\}$. Then as an algebra $A \cong E^{0} A$ and so $A$ may be bigraded (with the first gradation length). Therefore $H^{*}(A)=\operatorname{Ext}_{A} *(F, F)$ is trigraded. The set $\left\{a_{i}\right\}$ is a basis for the set of indecomposable elements of $A$, hence $H^{1, *, *}(A)=H^{1,1, *}(A)$ has basis $\left\{\alpha_{i}\right\}$ where $\alpha_{i} \in H^{1,1, q}(A)$ corresponds to $a_{i} \in A_{1, q}$. We say that $A$ is a homogeneous Koszul algebra if the cohomology algebra is generated by the set $\left\{\alpha_{i}\right\}$. This is equivalent to the condition $H^{s, p, q}(A)=0$ unless $s=p$. We say that a pre-Koszul algebra $A$ is a Koszul algebra if $E^{0} A$ is a homogeneous Koszul algebra.

(2.2) Examples of Koszul Algebras. (1) The tensor algebra $T\left\{x_{i}\right\}$, the free commutative algebra $A\left\{x_{i}\right\}$, and the exterior algebra $E\left\{x_{i}\right\}$ are homogeneous Koszul algebras.

(2) If $L$ is a graded Lie algebra then the universal enveloping algebra $U(L)$ is a Koszul algebra and any basis $\left\{a_{i}\right\}$ of $L$ is a Koszul set of generators. The defining relations are

$$
a_{i} a_{j}-(-1)^{\operatorname{deg} a_{i} \operatorname{deg} a_{j}} a_{j} a_{i}-\left[a_{i}, a_{j}\right]=0,
$$

and $E^{0} U(L)=A\left\{b_{i}\right\}$.

(3) If $L$ is a graded restricted Lie algebra and char $F=2$, then $V(L)$ is a Koszul algebra. The defining relations are those of (2) and $a_{i}^{2}+\xi\left(a_{i}\right)=0$ where $\xi$ is the restriction, $E^{0} V(L)=E\left\{b_{i}\right\}$ is the associated homogeneous Koszul algebra.

(4) The Steenrod algebra $A$ has Koszul generators $\left\{\beta P^{i}\right\} \cup\left\{P^{j}\right\}$, $i \geqq 0, j>0$ (if $p=2, P^{i}=\mathrm{Sq}^{i}$ and $\beta P^{i}$ omitted) subject to the Adem relations (and their Bocksteins). Since $P^{0}=1, A$ is not homogeneous.

(5) The Steenrod algebra $A_{L}$ for simplicial restricted Lie algebras $[4,7.1,8.8]$ has the same Koszul generators and relations as (4) except $P^{0}=0$. Therefore $A_{L}$ is isomorphic to the associated homogeneous Koszul algebra $E^{0} A$ of (4).

(2.3) Koszul resolutions. Let $A$ be a Koszul algebra with Koszul generators $\left\{a_{i}\right\}$. Let $R$ and $L$ be right and left $A$-modules respectively. Each element $x \in H_{p, p}\left(E^{0} A\right)$ may be uniquely represented by a $p$-cycle of $\bar{B}\left(E^{0} A\right)$ of the form $\sum_{i}\left[b_{i_{1}}|\cdots| b_{i_{p}}\right]$ where $b_{i_{j}} \in E_{1, *}^{0} A$ (there are no $(p+1)$-chains of length $p$ ). Hence the natural isomorphism $\iota: E_{1}^{0} A \stackrel{\approx}{\rightarrow} F_{1} I(A)$, induced by the splitting $1-\eta \epsilon: A \rightarrow I(A)$, ex- 
tends to a natural inclusion of modules

$$
\iota: R \otimes H_{*, *}\left(E^{0} A\right) \otimes L \rightarrow B(R, A, L)
$$

where $B(R, A, L)$ is the bar construction (denoted $B(R, L)$ in [3]). It is not difficult to show that Image $(\iota)$ is actually a subcomplex of $B(R, A, L)$. The Koszul complex $K_{*}(R, A, L)$ is then defined to be the complex whose module is $R \otimes H_{*, *}\left(E^{0} A\right) \otimes L$ and whose differential $d$ is determined by requiring $\iota$ to be a map of complexes.

To describe $d$ explicitly, suppose $x \in H_{p, p}\left(E^{0} A\right)$ is represented (as above) by $\sum_{i}\left[b_{i_{1}}|\cdots| b_{i_{p}}\right]$. Since

$$
\partial\left(\sum_{i}\left[b_{i_{1}}|\cdots| b_{i_{p}}\right]\right)=0 \text { in } \bar{B}\left(E^{0} A\right)
$$

it follows that in $\bar{B}(A)$

$$
\begin{aligned}
& \partial\left(\sum_{i}\left[a_{i_{1}}|\cdots| a_{i_{p}}\right]\right)=\sum_{i} \sum_{j=1}^{p-1}(-1)^{e_{j}}\left[a_{i_{1}}|\cdots| a_{i_{j}} a_{i_{j+1}}|\cdots| a_{i_{p}}\right] \\
&\left(e_{j}=\operatorname{deg}\left[a_{i_{1}}|\cdots| a_{i_{j}}\right]\right) \\
&=\sum_{i} \sum_{j=1}^{p-1} \sum_{k} f_{i_{j k}}\left[a_{i_{1}}|\cdots| a_{i_{j k}}|\cdots| a_{i_{p}}\right]
\end{aligned}
$$

for certain $f_{i_{j k}} \in F$ and $a_{i_{j k}} \in\left\{a_{i}\right\}$. Hence

$$
d(r \otimes x \otimes l)
$$

$$
\begin{aligned}
= & \sum_{m}(-1)^{\operatorname{deg} r} r a_{m} \otimes\left\{\sum_{i, i_{1}=m}\left[b_{i_{2}}|\cdots| b_{i_{p}}\right]\right\} \otimes l \\
& +(-1)^{\operatorname{deg} r} r \otimes\left\{\sum_{j=1}^{p-1} \sum_{i, k} f_{i_{j k}}\left[b_{i_{1}}|\cdots| b_{i_{j k}}|\cdots| b_{i_{p}}\right]\right\} \otimes l \\
& +\sum_{m}(-1)^{\operatorname{deg} r+e_{p}} r \otimes\left\{\sum_{i, i_{p}=m}\left[b_{i_{1}}|\cdots| b_{i_{p-1}}\right]\right\} \otimes a_{m} l .
\end{aligned}
$$

Proposition 1. There is a canonical injection of complexes $\iota: K_{*}(R, A, L) \rightarrow B_{*}(R, A, L)$ given by

$$
\iota\left(r \otimes\left\{\sum_{i}\left[b_{i_{1}}|\cdots| b_{i_{p}}\right]\right\} \otimes l\right)=r \otimes \sum_{i}\left[a_{i_{1}}|\cdots| a_{i_{p}}\right] \otimes l .
$$

If $R=F=L$ then $\iota$ is an injection of differential co-algebras.

The following is the main theorem of our theory:

Theorem 2. If $A$ is a Koszul algebra then $\operatorname{Tor}_{*, *}^{A}(R, L)$ is the homology of the Koszul complex $K_{*}(R, A, L)$. 
Sketch of the Proof. The filtration of $A$ induces a filtration of the bar construction $B(R, A, L)$. There results a spectral sequence $E_{*, *}^{1}=R \otimes H_{*, *}\left(E^{0} A\right) \otimes L \Rightarrow \operatorname{Tor}_{*, *}(R, L)$ whose $\left(E^{1}, d^{1}\right)$-term is readily seen to be the Koszul complex $K_{*}(R, A, L)$. Since $A$ is Koszul, $H_{s, p}\left(E^{0} A\right)=0$ for $s \neq p$ and so the sequence collapses $\left(E^{2}=E^{\infty}\right)$.

Since $\operatorname{Tor}^{A}(R, L)=L$ it is clear that $K_{*}(A, A, L)$ is a resolution of $L$ by free left $A$-modules. We call $K_{*}(A, A, L)$ the Koszul resolution of $L$. Similarly $K_{*}(R, A, A)$ is the Koszul resolution of $R$ (by free right $A$-modules).

Let $K^{*}(R, A, L)$ denote the graded $F$-dual of $K_{*}(R, A, L)$. Then $K^{*}(R, A, L)$ is called the co-Koszul complex for computing $\operatorname{Ext}_{A}\left(L, R^{*}\right)\left(=\operatorname{Tor}^{A}(R, L)^{*}\right)$.

For simplicity we shall limit our discussion here to the case $R=F=L$. Since $K_{*}(F, A, F)$ is a differential co-algebra it follows that $K^{*}(F, A, F)$ is a differential algebra. It is important to note that $K^{*}(F, A, F)$ admits an explicit set of generators and relations defined in terms of the Koszul generators and relations of $A$.

Since $A$ is generated by $\left\{a_{i}\right\}$ we can choose an $F$-basis $B_{A}$ for $A$ consisting of $1, a_{i}$, and certain monomials $a_{i_{1}} \cdots a_{i_{n}}, a_{i_{j}} \in\left\{a_{i}\right\}$. We can also choose a set $S \subset U=\bigcup_{n=1}^{\infty} I_{1} \times \cdots \times I_{n}$ (where each $I_{j}=I$ is the indexing set of $\left\{a_{i}\right\}$ ) such that for each $a \in B_{A}-\{1\}$ there is a unique $\left(i_{1}, \cdots, i_{n}\right) \in S$ such that $a=a_{i_{1}} \cdots a_{i_{n}}$. We can assume the relations of $A$ are written in admissible form with respect to $B_{A}$ and $S$, i.e.,

$$
a_{k} a_{l}=\sum_{m} f\left(\begin{array}{c}
k, l \\
m
\end{array}\right) a_{m}+\sum_{(i, j) \in S} f\left(\begin{array}{l}
k, l \\
i, j
\end{array}\right) a_{i} a_{j}
$$

where $f\left(\begin{array}{l}\boldsymbol{k}, l \\ m\end{array}\right)$ and $f\left(\begin{array}{l}\boldsymbol{k}, l, l \\ i, j\end{array}\right)$ are in $F$. Let $B_{A}^{*}=\left\{1, \alpha(i), \alpha\left(i_{1}, \cdots, i_{n}\right)\right\}$ denote the dual basis, i.e., if $\left(i_{1}, \cdots, i_{n}\right) \in S$ then $\left\langle\alpha\left(i_{1}, \cdots, i_{n}\right), a\right\rangle=1$ if $a=a_{i_{1}} \cdots a_{i_{n}}$ and zero otherwise. A basis $B_{E^{0} A_{A}}$ for $E^{0} A$ is formally obtained from $B_{A}$ by replacing the letter $a$ with the letter $b$ and similarly $B_{E^{0} \boldsymbol{A}}$ from $B_{\boldsymbol{A}}^{*}$ by replacing $\alpha$ with $\beta$. Admissible relations for $E^{0} A$ are

$$
b_{k} b_{l}=\sum_{(i, j) \in S} f\left(\begin{array}{l}
k, l \\
i, j
\end{array}\right) b_{i} b_{j} .
$$

Let $\beta_{i}$ denote the class of $[\beta(i)]$ in $H^{1,1, *}\left(E^{0} A\right)$.

Theorem 3. Let $A$ be a Koszul algebra with Koszul generators $\left\{a_{i}\right\}$ and admissible relations (2.5). Then

(1) The cohomology algebra $H^{*}(A)$ is the homology of the co-Koszul complex $K^{*}(F, A, F)=H^{*}\left(E^{0} A\right)$. 
(2) As an algebra, $K^{*}(F, A, F)$ is generated by $\left\{\beta_{i}\right\}$ subject to the relations: if $(i, j) \in S$ then

$$
(-1)^{n i, j} \beta_{i} \beta_{j}+\sum_{(k, l) \in U-S}(-1)^{v_{k}, l f}\left(\begin{array}{l}
k, \eta \\
i, j
\end{array}\right) \beta_{k} \beta_{l}=0
$$

where $\nu_{u, v}=\operatorname{deg} \beta_{u}+\left(\operatorname{deg} \beta_{u}-1\right)\left(\operatorname{deg} \beta_{v}-1\right)$. The differential is determined by

$$
\delta \beta_{m}=\sum_{(k, l) \in U \rightarrow S}(-1)^{v k, l f}\left(\begin{array}{c}
k, l \\
m
\end{array}\right) \beta_{k} \beta_{l} .
$$

Sketch of Proof. Part (1) follows from Theorem 2 and duality. Now $A$ is a Koszul algebra and so $H^{*}\left(E^{0} A\right)$ is generated by $\left\{\beta_{i}\right\}$. If $\beta(i, j) \in B_{E^{0} A}^{*}$ then

$$
\begin{aligned}
\delta[\beta(i, j)]= & (-1)^{v_{i, j}, j}[\beta(i) \mid \beta(j)] \\
& +\sum_{(k, l) \in U-S}(-1)^{v_{k}, l f}\left(\begin{array}{l}
k, l \\
i, j
\end{array}\right)[\beta(k) \mid \beta(l)]
\end{aligned}
$$

in $\bar{C}^{*}\left(E^{0} A\right)$ and so the desired relations hold. The derivation of the differential is similar and is dual to $d$ of (2.4).

3. Applications. (1) Graded Lie algebras. If $L$ is a graded Lie algebra then $U(L)$ is a Koszul algebra (see (2.2) Example (2)) and $E^{0} U(L)=E\left(L^{-}\right) \otimes A\left(L^{+}\right)$, where $L^{+}, L^{-} C L$ are the elements of even and odd dimension respectively, with the convention that $L^{+}=L$ and $L^{-}=\varnothing$ if char $F=2$. It is classical that $H_{*} A\left(L^{+}\right)=E\left(L^{+}\right)$ and $H_{*} E\left(L^{-}\right)=\Gamma\left(L^{-}\right)$the divided polynomial algebra. Hence $K_{*}(F, U(L), U(L))=\Gamma\left(L^{-}\right) \otimes E\left(L^{+}\right) \otimes U(L)$ and Proposition 1 shows that this resolution coincides with that described by May [3, p. 131].

(2) Graded restricted Lie algebras (char $F=2$ ). Let $L$ be a graded restricted Lie algebra and assume char $F=2$. Then $V(L)$ is a Koszul algebra (see (2.2) Example (3)) and $E^{0} V(L)=E(L)$. The embedding of Proposition 1 shows that $K_{*}(F, V(L), V(L))=\Gamma(L) \otimes V(L)$ agrees with May's resolution $X(L)$ [3, Remarks 10, p. 140].

(3) The Steenrod algebra. Let $A$ denote the Steenrod algebra and for simplicity let $p=2$ (analogous results hold for $p$ odd). Now $A$ is a Koszulalgebra (see (2.2) Examples (4) and (5) and $E^{0} A \cong A_{L}$. Applying Theorem 3 we see that $K^{*}\left(Z_{2}, A, Z_{2}\right)$ is the differential algebra generated by $\left\{\sigma_{i}\right\}, i>0\left(\sigma_{i}\right.$ dual to $\left.\mathrm{Sq}^{i}\right)$ subject to the relations: if $a \geqq 2 b>0$

$$
\sigma_{a} \sigma_{b}=\sum_{j=2 b}^{\{2(a+b) / 3\}}\left(\begin{array}{c}
a-j-1 \\
j-2 b
\end{array}\right) \sigma_{j} \sigma_{a+b-j}
$$


with differential

$$
\delta \sigma_{a}=\sum_{j=1}^{[2 a / 8\}}\left(\begin{array}{c}
a-j-1 \\
j
\end{array}\right) \sigma_{j} \sigma_{a-j}
$$

where $\{x\}$ is the greatest integer less than $x$.

Let $\left(E^{1} S, d^{1} S\right)$ be the $\left(E^{1}, d^{1}\right)$-term of the Adams spectral sequence (derived from the lower 2-central series filtration) for the simplicial sphere spectrum FS [1]. It is easy to check that there is an anti-automorphism of differential $Z_{2}$-algebras

$$
\Phi:\left(K^{*}\left(Z_{2}, A, Z_{2}\right), \delta\right) \rightarrow\left(E^{1} S, d^{1} S\right)
$$

extending the map of generators given by $\sigma_{i+1} \rightarrow \lambda_{i}(i \geqq 0)$.

\section{REFERENCES}

1. A. K. Bousfield, E. B. Curtis, D. M. Kan, D. G. Quillen, D. L. Rector and $\mathrm{J}$. W. Schlesinger, The mod-p lower central series and the Adams spectral sequence, Topology 5(1966), 331-342, MR 33 \#8002.

2. H. Cartan and S. Eilenberg, Homological algebra, Princeton Univ. Press, Princeton, N. J., 1956. MR 17, 1040.

3. J. P. May, The cohomology of restricted Lie algebras and of Hopf algebras, J. Algebra 3 (1966), 123-146. MR 33 \#1347.

4. S. B. Priddy, Primary cohomology operations for simplicial Lie algebras, Illinois J. Math. (to appear).

5. - Koszul resolutions, Trans. Amer. Math. Soc. (to appear).

Northwestern University, Evanston, Illinois 60201 